\title{
THE INFLUENCE OF SILVER NANOPARTICLES SYNTHESIS ON THEIR PROPERTIES
}

\author{
Anna MražÍKováa, ${ }^{a *}$, Oksana Velgosováa ${ }^{a}$, Jana Kavuličová ${ }^{b}$, \\ Stanislav KRuM ${ }^{c}$, JaroslaV MáleK ${ }^{c}$ \\ ${ }^{a}$ Institute of Materials and Quality Engineering, Faculty of Materials, Metallurgy and Recycling, Technical \\ University of Kosice, Slovakia \\ ${ }^{b}$ Institute of Metallurgy, Faculty of Materials, Metallurgy and Recycling, Technical University of Kosice, Slovakia \\ ${ }^{c}$ Department of Materials Engineering, Faculty of Mechanical Engineering, Czech Technical University in \\ Prague, Czech Republic \\ * corresponding author: anna.mrazikova@tuke.sk
}

ABSTRACT. Application of green methods to replace physical and chemical methods for synthesis of silver nanoparticles (AgNPs) has become necessary not only from economic aspect but especially due to its significant impact on ecosystem. The properties of biologically synthesized AgNPs using green algae Parachlorella kessleri (P. kessleri) and chemically prepared were investigated and compared. The UVvis analysis confirmed a high stability of biosynthesized AgNPs as well as chemically synthesized gelatin modified citrate-AgNPs. Scanning electron microscopy (SEM) and Transmission electron microscopy (TEM) revealed different sizes and shapes of AgNPs synthesized in different ways. Biosynthesized AgNPs have similar inhibitory antimicrobial activity as gelatin/sodium citrate-AgNPs.

KEYWORDS: silver nanoparticles; biosynthesis; chemical reduction; gelatin; anti-microbial activity.

\section{INTRODUCTION}

In recent years the use of noble metal nanomaterials in many industrial applications including physics, chemistry, electronics, optics, material science has rapidly increased. Furthemore, silver containing materials have gained a great attention especially due to their antimicrobial properties. Therefore, AgNPs are now being used to reduce infections, to prevent biofilm formation on protheses, catheters, dental materials and also on stainless steel materials [1 5$]$.

Physical and chemical methods generally used for AgNPs synthesis very often involved toxic chemicals that can contaminate the nanoparticles 6]. Such nanoparticles are released into environment in different stages of their production, apllication and even disposal of nanowastes what can consequently lead to contamination of the whole ecosystem. Finally, the majority of nanoparticles accumulates in fresh and marine ecosystems [7]. Oukarroum et al. [8] in their reports outlined a negativelly effect of such AgNPs on both freshwater and marine algae by strong decrease in viable algal cells. Therefore there is a need to replace physical and chemical techniques of AgNPs preparation by green alternatives, which are cost-effective, safe, environment-friendly and easily scaled up for large syntheses of NPs. The use of biomolecules like proteins and lipids present on NPs surfaces has a great potential in AgNPs synthesis due to their non-toxic nature and also gentle synthetic procedures [9, 10]. Therefore there is a growing concern to apply biomimetic which use plants, bacteria, fungi, yeast, actinomycetes and algae for synthesis of nanostructures of biocompatible metals and semicon- ductors [5].

The common problem of AgNPs application typically prepared via reduction of a silver precursor using chemical or physical means is dispersion instability against aggregation. One of the possibilities to enhance stabilization of nanoparticles is addition of surface-protecting agents such as organic ligands namely chitosan, polysacharides and gelatin or inorganic capping materials [11-13]. Lee [12] and Sivera [13] reported the gelatin-modified AgNPs exhibited long-term stability against aggregation and maintained unchanged optical and physical properties and a high antibacterial activity for several months at ambient temperature. The similar properties were also observed in biologically synthesized AgNPs. Functional groups of the biological materials are responsible for the reduction of the $\mathrm{Ag}^{+}$and subsequent stabilization of nanoparticles 9, 14, 15. Green algae are well-known by biomass containing different organic biologically active compounds such as chlorophylls, carotenoids, flavonoids, proteins, vitamins and minerals [5]. Natural polymer on algae is considered to be suitable for stabilization of inorganic silver nanoparticles.

We have recently reported that AgNPs biosynthesized using $P$. kessleri showed long-term stability at the higher $\mathrm{pH}$ values 16 . The novelty of this work is investigation and comparison of properties biologically synthesized AgNPs using the algae P. kessleri and chemically synthesized AgNPs. Chemically synthesized AgNPs were performed by two different ways: using sodium citrate and both sodium citrate and gelatin as a reducing and capping agent. This study 

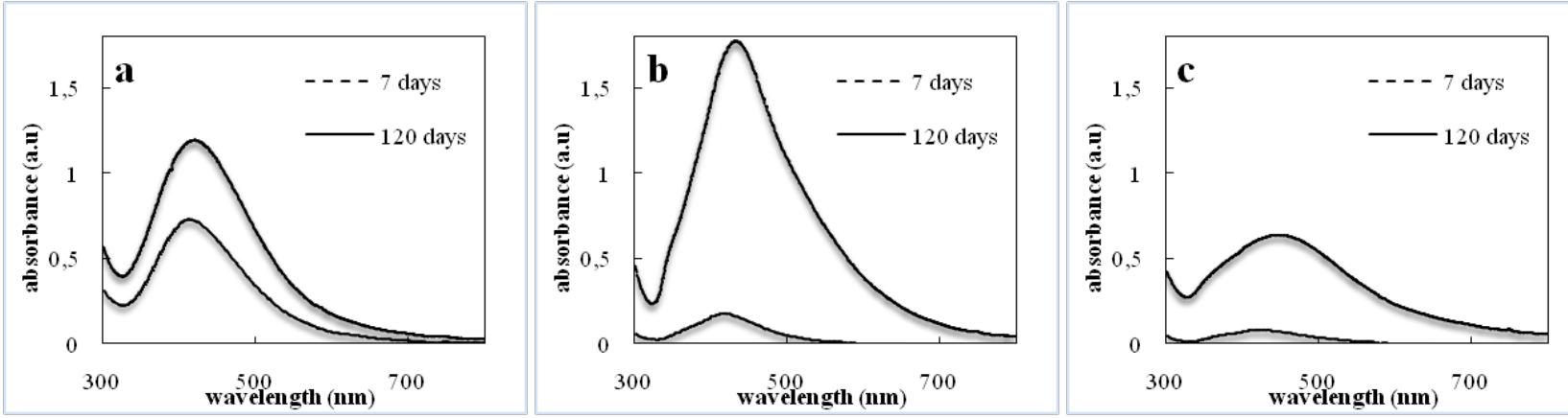

Figure 1. UV-vis spectra of (a) biosynthesized AgNPs (b) gelatin/sodium citrate-AgNPs (c) sodium citrate-AgNPs.

also compares the antimicrobial activities of AgNPs against the algae $P$. kessleri.

\section{MAterials AND Methods}

\subsection{SyNTHESIS OF SILVER NANOPARTICLES}

The green algae $P$. kessleri were cultivated on agar plates in Petri dishes for 3 weeks at the ambient temperature and light mode $(12: 12)$. The extract was filtered and the filtrate was centrifuged at $9000 \mathrm{rpm}$ for $15 \mathrm{~min}$ and supernatant was added into Erlenmeyer flasks containing $250 \mathrm{ml}$ of $\mathrm{AgNO}_{3}$ solution $(0.29 \mathrm{mM}$ ) and used for biosynthesis of AgNPs. The Erlenmayer flasks were stored under lighting condition at the ambient temperature to allow reducing the silver ions into AgNPs.

Chemically synthesized AgNPs were prepared using chemical reduction method [7, 17]. The $15 \mathrm{ml}$ of sodium citrate (0.5 wt. \%) solution as a reducing agent was added drop by drop to the $250 \mathrm{ml}$ of aqueous $\mathrm{AgNO}_{3}(0.29 \mathrm{mM})$. In the case of chemically synthesized AgNPs using mixed gelatin/sodium citrate, first of all, to prepare stock solution of $\mathrm{AgNO}_{3}$, the gelatin (0.01 wt.\%) was dispersed in $250 \mathrm{ml}$ of $0.29 \mathrm{mM}$ of $\mathrm{AgNO}_{3}$ to prevent particle agglomeration. Silver nanoparticles were prepared by adding drop-wise of $15 \mathrm{ml}$ of (0.5 wt.\%) sodium citrate solution into $\mathrm{AgNO}_{3}$ solution. Both solutions of chemically prepared AgNPs were stirred at $700 \mathrm{rpm}$ with a magnetic stirring bar at $70^{\circ} \mathrm{C}$ for 30 minutes. Erlenmayer flasks were stored in lighting condition at the ambient temperature and the end point of the reaction was the appearance of pale yellow-brown and dark brown colour.

\subsection{Antimicrobial assay}

The ability of AgNPs to inhibit the formation of algae biofilm was performed by standard diskdiffusion method [18]. The $1 \mathrm{ml}$ of algal suspension $(105 \mathrm{CFU} / \mathrm{ml})$ was used to seed agar plates consisted of $2 \%$ agar and culture medium (Milieu Bristol). The $25 \mathrm{\mu l}$ of colloidal sollutions AgNPs were added to sterile swabs $(6 \mathrm{~mm})$ placed on agar plates seeded with microorganisms. The minimum inhibitory concentration (MIC) was read after 7 days of incubation at the ambient temperature and light mode (12:12).

\subsection{Characterizations}

The nanoparticle colloidal solutions were stirred with magnetic stirrer with heating (IKA C-MAG HS4).

The absorbance of the AgNPs dispersions was analyzed using an UV-vis spectra from 300 to $800 \mathrm{~nm}$ with UNICAM UV/vis Spectrometer UV4. The absorbance was recorded on days 7 and 120 .

Transmission Electron Microscope (TEM; JEM2000FX, JEOL) at $200 \mathrm{kV}$ was used to determine the size and morphology of AgNPs on day 120.

Scanning electron Microscopy (SEM) analysis was done using JEOL JSM-7600F and used to determine the surface morphology properties on day 120 .

$\mathrm{EVE}^{\mathrm{TM}}$-NanoEnTek was used to automate cell counting by the standard trypan blue technique.

The observation of algae cells eradication on agar plates was done by macroscope LEICA WILD M32.

\section{RESUlts AND DISCUSSION}

The formation of silver nanoparticles synthesized either chemically and biologically was clearly observed after 3 and 24 hours reaction time by solution colour changes and confirmed by UV-vis spectroscopy as depicted in Fig. 1. In the case of P. kessleri the solution colour changed from pale yellow to yellow-brown. In the presence of citrate and gelatin/sodium citrate the solution colour changed to pale yellow-brown and dark brown, respectively. The UV-vis measurements showed the increase of the absorption maximum within 120 days in all three nanoparticle samples. This indicated the formation of AgNPs in the solution at the time. The biologically synthesized nanoparticles exhibited an increase of the broad absorption band on day 120 and only minor shift of UV-visible spectrum (Fig. 17), what indicated a long-term stability of the silver nanoparticles [19]. Kadukova [15] reported that AgNPs produced by P. kessleri can be stable even more than 6 months. The AgNPs chemically produced using gelatine as a capping agent exhibited the most significant deviation at increase of the absorption maximum at $433 \mathrm{~nm}$ on day 120 (Fig. 1p). As reaction time increased more amine residues of gelatine were being released into the reaction system and consequently, reduction of silver ions slowly proceeded [12]. Shift of UV-visible spectrum (Fig. 1p) 

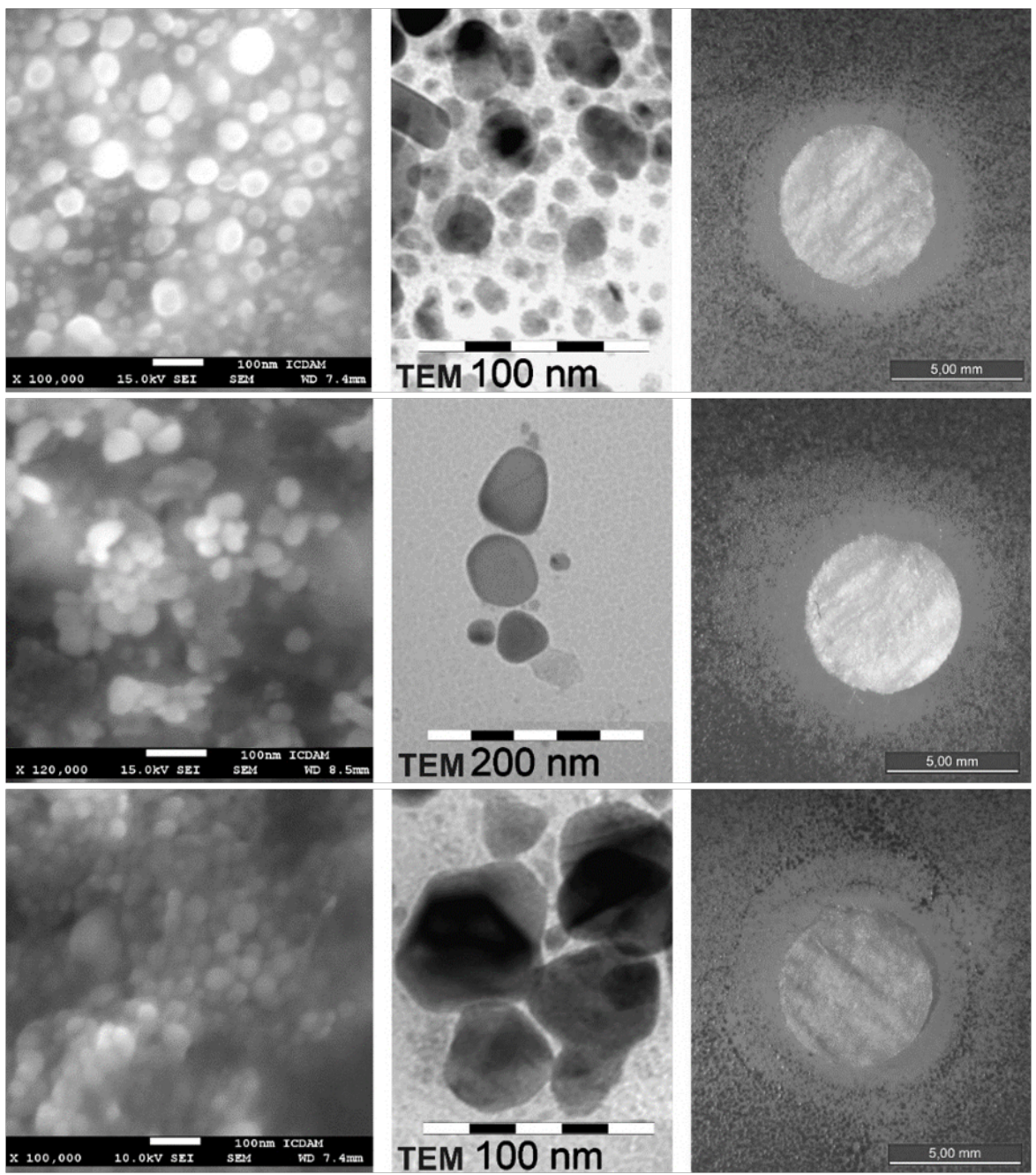

Figure 2. SEM and TEM images and the antimicrobial effect of (a,top) biosynthesized AgNPs (b,middle) gelatin/sodium citrate-AgNPs (c,bottom) sodium citrate-AgNPs.

from 416 to $433 \mathrm{~nm}$ points to the creation of larger average particle sizes as it was reported by many authors [19, 20]. For AgNPs obtained and stabilized only using sodium citrate the UV-vis increase of the absorption maximum on day 120 was not as strong as in the case of biologically synthesized AgNPs and gelatin modified sodium citrate-AgNPs as well. The most significant shift of UV-vis absorption maximum from day 7 to 120 (from 360 to $450 \mathrm{~nm}$ ) was observed in the solution with citrate-AgNPs without addition of capping agents. Such broadening and shift of SPR indicate the presence of larger nanoparticle sizes than above mentioned and also showed their short-term stability [20].

The occurrence of symmetrical sharp UV-vis absorption peaks typically located around $400 \mathrm{~nm}$ observed on day 120 in the solutions with biologically synthesized AgNPs as well as gelatin/sodium citrate-AgNPs (Fig. 1 $\mathrm{ab}$ ) indicated the presence of stable nanoparticles. The broad absorption band observed in citrateAgNPs, (Fig. 1k) indicated much less uniform and stabilized nanoparticles. The SEM and TEM micrographs (Fig. 2f) obtained after 120 days revealed the presence of large particle sizes (from 7 to $85 \mathrm{~nm}$ ) and also small agglomerates and some dispersed AgNPs. It is very likely that agglomeration was caused by diminishing electrostatic repulsion [21]. Based on our results the addition of gelatin to $\mathrm{Ag}^{+}$solution in the process of AgNPs formation improved stability and dispersibility of nanoparticles. Biomolecules like peptides and proteins present in gelatin are able readily interact with metals and hydrophilic ligands protect gelatin coated AgNPs in aqueous solution. The coating serves to provide proper gap between the silver core $[12,13]$.

The silver nanoparticles, which were reached using biological approaches, showed on SEM and TEM micrographs (Fig. 2a) spherical particles with average particle size of $15 \mathrm{~nm}$. The role of active compounds of biomass responsible for the process of AgNPs formation and stabilization was confirmed by the work of several authors [6, 15, 23. Their results indicated that the various functional groups, especially amine, carboxyl, sulphydril and hydroxyl moieties present in the proteins, primarily cause the reduction of nanoparticles. Formation of smaller sizes 
gelatin/sodium citrate-AgNPs (from 4 to $55 \mathrm{~nm}$ ) might be caused by presence of a higher gelatin concentration as demonstrated 14, 22. SEM and TEM images (Fig. 2p) of gelatin-protected particles also revealed the nanoparticles of different spherical and pyramidal shapes.

Antimicrobial effects of biosynthesized and chemically synthesized AgNPs against the green algae P. kessleri were observed in all three cases. The results revealed that chemically synthesized sodium citrateAgNPs caused only particular inhibition (Fig. 2.) against algae. Double zone of inhibition observed around the swabs impreganated with sodium citrateAgNPs was attributable to their bigger sizes, where AgNPs were not able to pass through the pores on the cell wall. Such aggregate formation might act as a binding agent between cells and inhibited algal cells growth [8]. Stronger extent of algae cells eradication and clear circular inhibition zone was observed around the swabs impregnated with gelatin/sodium citrate-AgNPs and biosynhesized AgNPs (Fig. 2ab). According to literature [3, 14, the nanoparticles of smaller sizes have a higher antibiofilm activity due to the largest surface/volume ratio what is most easily to reach cellular proximity. Such AgNPs cause structural changes and damages of cellular membrane that lead to cell death [3]. Our results indicated that biosynthesized AgNPs have similar inhibitory antimicrobial activity as gelatin/sodium citrate-AgNPs against biofilm formation and owing to their easy and inexpansive synthesis appear to be good alternative to chemically prepared AgNPs.

\section{Conclusion}

Silver nanoparticles were synthesized bio- and chemical reduction of $\mathrm{Ag}^{+}$ions. The UV-vis spectroscopy revealed that the addition of gelatin positively affected size and long-term stability of chemically synthesized citrate-AgNPs. Gelatin coated citrate-AgNPs also displayed enhanced antialgal effects in comparison with citrate-AgNPs. The UV-vis, SEM and TEM analyses revealed that biosynthesized AgNPs using algae extract exhibited long-term stability and also good antimicrobial activity against the green algae which could be attributed to the smallest sizes of the AgNPs. The extract from the green algae P. kessleri can adequately act as both reducing and capping agents. The results implied that biosynthesized AgNPs can be good alternative for preparation of materials which inhibit the biofilm formation.

\section{ACKNOWLEDGEMENTS}

This work was financially supported by Slovak Grant Agency (VEGA 1/0134/19).

\section{REFERENCES}

[1] Zhang X., Wang H., Li J., He X., Hang R., Yang Y., Tang B.: The fabrication of Ag-containing hierarchical micro/nano-structure on titanium and its antibacterial activity. Mater. Lett. 193, 2017, p. 97-100. DOI:10.1016/j.matlet.2017.01.094

[2] Guzmán M. G., Dille J., Godet S.: Synthesis of silver nanoparticles by chemical reduction method and their antibacterial activity. Int. J. of Chemical and Biomolecular Eng., 2:3, 2009, p. 104-111. DOI:10.1016/j.nano.2011.05.007

[3] Inbakandan D., Kumar C., Abraham L. S., Kirubagaran R., Venkatesan R., Khan S.A.: Silver nanoparticles with anti microfouling effect: A study against marine biofilm forming bacteria. Colloids and Surfaces B: Biointerfaces 111, 2013, p. 636-643. DOI:10.1016/j.colsurfb.2013.06.048

[4] Oluwafemi O. S., Vuyelwa N., Scriba M., Songca S. P.: Green controlled synthesis of monodispersed, stable and smaller sized starch-capped silver nanoparticles. Mater. Lett. 106, 2013, p. 332-336. DOI:10.1016/j.matlet.2013.05.001

[5] Shankar P.D., Shobana S., Karuppusamy I., Pugazhendhi A., Ramkumar V.S., Arvindnarayan S., Kumar G.: A review on the biosynthesis of metallic nanoparticles (gold and silver) using bio-components of microalgae: Formation mechanism and applications. Enz. and Microb. Technol. 95, 2016, p. 28-44. DOI:10.1016/j.enzmictec.2016.10.015

[6] Bogireddy N. K. R., Kumar H. A. K., Mandal B. K.: Biofabricated silver nanoparticles as green catalyst in the degradation of different textile dyes J. of Environ. Chem. Eng. 4, 2016, p. 56-64. DOI:10.1016/j.jece.2015.11.004

[7] Girilal M., Krishnakumar V., Poornima P., Fayaz A.M., Kalaichelvan P.T.: A comparative study on biologically and chemically synthesized silver nanoparticles induced Heat Shock Proteins on fresh water fish Oreochromis niloticus. Chemosphere, 139, 2015, p.461-468. DOI:10.1016/j.chemosphere.2015.08.005

[8] Oukarroum A., Bras S., Perreault F., Popovic R.: Inhibitory effects of silver nanoparticles in two green algae, Chlorella vulgaris and Dunaliella tertiolecta. Ecotoxicology and Environ. Safety, 78, 2012, p. 80-85. DOI:10.1016/j.ecoenv.2011.11.012

[9] Sharma D., Kanchi S., Bisetty K.: Biogenic synthesis of nanoparticles: A review. Arabian J. of Chemistry. 2015. DOI:10.1016/j.arabjc.2015.11.002

[10] Srinithia B., Kumar V.V., Vadivel V., Pemaiah B., Anthony S. P., Mathuraman M. S.: Synthesis of biofunctionalized AgNPs using medicinally important Sida cordifolia leaf extract for enhanced antioxidant and anticancer activities. Mater. Lett. 170, 2016, p. 101-104. DOI:10.1016/j.matlet.2016.02.019

[11] Abdulla-Al-Mamun M., Kusumoto Y., Muruganandham M.: Simple new synthesis of copper nanoparticles in water/acetonitrile mixed solvent and their characterization. Mater. Lett. 63, 2009, p. 2007-2009. DOI:10.1016/j.matlet.2009.06.037

[12] Lee Ch. and Zhang P.: Facile synthesis of gelatin-protected silver nanoparticles for SERS applications. J. Raman Spectrosc. 44, 2013, p. 823-826. DOI:10.1002/jrs.4304 
[13] Sivera M., Kvitek L., Soukupova J., Panacek A., Prucek R., Vecerova R., Zboril R.: Silver Nanoparticles Modified by Gelatin with Extraordinary pH Stability and Long-Term Antibacterial Activity. PLOS ONE, 9(8) e103675, 2014. DOI:10.1371/journal.pone.0103675

[14] Ethiraj A. S., Jayanthi S., Ramalingam Ch., Benerjee Ch.: Control of size and antimicrobial activity of green synthesized silver nanoparticles. Mater. Lett. 185, 2016, p. 526-529. DOI:10.1016/j.matlet.2016.07.114

[15] Kadukova J.: Surface sorption and nanoparticle production as a silver detoxification mechanism of the freshwater alga Parachlorella kessleri Biores. Tech. 216, 2016, p. 406-413. DOI:10.1016/j.biortech.2016.05.104

[16] Velgosová O., Mražíková A., Marcinčáková R.: Influence of $\mathrm{pH}$ on green synthesis of $\mathrm{Ag}$ nanoparticles. Mater. Lett. 180, pp. 336-339 (2016). DOI:10.1016/j.matlet.2016.04.045

[17] Sileikaite A., Prosycevas I., Puiso J., Juraitis A., Guobiens A.: Analysis of Silver Nanoparticles Produced by Chemical Reduction of Silver Salt Solution. Materials Science (Medziagotyra) 12(4) 2006, p. 287-291.

[18] Kavita K., Singh V.K., Jha B.: 24-Branched $\Delta 5$ sterols from Laurencia papillosa red seaweed with antibacterial activity against human pathogenic bacteria. Microbial Research. 169 (4), 2014, p. 301-306. DOI:10.1016/j.micres.2013.07.002
[19] Villanueva-Ibáñez M., Yañez-Cruz M.G., R. ÁlvarezGarcía R., Hernández-Pérez M.A., Flores-González M.A.: Aqueous corn husk extract - mediated green synthesis of $\mathrm{AgCl}$ and $\mathrm{Ag}$ nanoparticles Mater. Lett. 152, 2015, p. 166-169. DOI:10.1016/j.matlet.2015.03.097

[20] Rashid M.U., Bhuiyan M.K.H., Quayum M.E.: Synthesis of Silver Nano Particles (Ag-NPs) and their uses for Quantitative Analysis of Vitamin C Tablets. J. Pharm. Sci. 12, 2013, p. 29-33. DOI:10.3329/dujps.v12i1.16297

[21] Roh J., Umh H. N., Sim J., Park S., Yi J., Kim Y.: Dispersion stability of citrate- and PVP-AgNPs in biological media for cytotoxicity test Korean. J. Chem. Eng. 30 (3), 2013, p.671-674. DOI:10.1007/s11814-012-0172-3

[22] Pootawang P., Saito N., Takai O.: Ag nanoparticle incorporation in mesoporous silica synthesized by solution plasma and their catalysis for oleic acid hydrogenation. Mater. Lett. 65, 2011, p. 1037-1040. DOI:10.1016/j.matlet.2011.01.009

[23] Patel V., Berthold D., Puranik P., Gantar M.: Screening of cyanobacteria and microalgae for their ability to synthesize silver nanoparticles with antibacterial activity. Biotechnol. Reports. 5, 2015, p. 112-119. DOI:10.1016/j.btre.2014.12.001 